\title{
Sono e doenças pulmonares crônicas: pneumopatias intersticiais difusas, asma brônquica e DPOC
}

\author{
Sleep and chronic lung diseases: diffuse interstitial lung diseases, \\ bronchial asthma, and COPD
}

Marília Montenegro Cabral, Paulo de Tarso Mueller

\begin{abstract}
Resumo
As doenças pulmonares crônicas podem ser agravadas por inúmeros fatores e comorbidades, inclusive pelos distúrbios respiratórios do sono. Embora alterações na qualidade de vida de pacientes com doenças pulmonares crônicas sejam normalmente determinadas a partir de variáveis diurnas, as alterações fisiopatológicas no sono pioram a qualidade de sono e interferem na história natural dessas doenças. Alterações da arquitetura do sono parecem ser um mecanismo comum entre essas doenças. Durante o sono, as vias respiratórias inferiores estão mais interligadas às vias respiratórias superiores, quando as alterações de resistências das vias aéreas superiores durante o sono somam-se às graves alterações resistivas de vias aéreas inferiores devido a asma e DPOC. Além disso, surgem complexas interações mecânicas e ventilatórias. 0 reconhecimento dessas interações possibilita uma melhor avaliação das exacerbações e da evolução dessas doenças.
\end{abstract}

Descritores: Sono; Asma; Doença pulmonar obstrutiva crônica; Doenças pulmonares intersticiais.

\begin{abstract}
Chronic lung diseases can be aggravated by various factors and comorbidities, including sleep-disordered breathing. Although changes in the quality of life of patients with chronic lung disease are usually related to daytime variables, the physiological changes in sleep impair the quality of sleep and interfere with the natural history of the disease. Alterations in sleep architecture appear to be a common mechanism in these diseases. During sleep, the upper and lower airways are more interconnected: changes in upper airway resistance during sleep are added to the severe resistive alterations in the lower airways due to asthma and COPD. In addition, there are complex mechanical and ventilatory interactions. The recognition of these interactions allows better assessment of the exacerbations and the progression of chronic lung diseases.
\end{abstract}

Keywords: Sleep; Asthma; Pulmonary disease, chronic obstructive; Lung diseases; interstitial.

\section{Introdução}

A fisiologia respiratória durante o sono é consideravelmente diferente quando comparada à vigília. Em indivíduos saudáveis, a passagem da vigília ao sono está associada à queda de 5-10\% da ventilação minuto, com queda adicional de até 40\% durante o sono REM. Vários são os fatores responsáveis pela queda da ventilação durante 0 sono em indivíduos saudáveis: redução da taxa metabólica basal, aumento da resistência da via aérea superior, perda do estímulo da vigília para respirar e fatores relacionados ao sono REM: diminuição do estímulo central muscular respiratório e hipotonia da musculatura acessória e intercostal da respiração. ${ }^{(1)}$

Essas alterações fisiológicas que ocorrem normalmente durante o sono não promovem efeitos deletérios em indivíduos saudáveis. Entretanto, em portadores de doenças pulmonares, especialmente em portadores de
DPOC, podem ocorrer acentuada hipoxemia e hipercapnia. ${ }^{(2)}$

Este capítulo pretende descrever as alterações fisiopatológicas que ocorrem durante o sono nas principais doenças pulmonares: pneumopatias intersticiais difusas, asma e DPOC.

\section{Pneumopatias intersticiais difusas}

As doenças pulmonares intersticiais formam um grupo heterogêneo com mais de 150 distúrbios em graus variados de inflamação e fibrose do parênquima e interstício pulmonar. A relação mútua entre o sono e essas doenças foi poucas vezes estudada. Existem evidências de baixa qualidade de sono que se associam à baixa qualidade de vida nesses pacientes. A fadiga é uma queixa frequente. Existe unanimidade pela alteração da arquitetura do sono, com redução 
da eficiência do sono, aumento proporcional do estágio 1 , redução do sono REM com latência prolongada e maior número de despertares. A redução de sono de ondas lentas também foi observada. ${ }^{(3)}$

Causas não respiratórias de alterações da arquitetura do sono são especificamente associadas a algumas dessas doenças, como na esclerose sistêmica, onde ocorrem discinesia esofagiana e refluxo, além de alta prevalência de movimentos periódicos das pernas. ${ }^{(4)}$

Entre as causas respiratórias, foi observado em dois estudos que a frequência de SAOS estava aumentada entre pacientes com doenças intersticiais pulmonares em relação a controles, mas os pacientes eram referenciados para 0 laboratório com sintomas sugestivos de SAOS e eram obesos, em média. Porém, a magnitude da função pulmonar em um desses estudos (CPT e DLCO) se correlacionou significativamente com $1 \mathrm{AH} .{ }^{(3)}$ Evidências atuais, em um estudo controlado, reforçam a alta prevalência de SAOS (88\%) associada à fibrose pulmonar idiopática, sugerindo que possivelmente, nessa doença, exista um sério comprometimento no controle neuromuscular da faringe pela própria doença em si ou pelo uso de corticosteroides sistêmicos. ${ }^{(5)}$

Com relação à dessaturação da hemoglobina durante o sono, quando sem queixas ou evidências de SAOS, não se constata quedas maiores que $3 \%$, o que parece ser muito contrastante com as condições de alta velocidade de fluxo sanguíneo capilar alveolar durante o exercício, onde há em geral dessaturação importante. Apesar disso, observou-se que uma saturação periférica em repouso em vigília $<90 \%$ é um fator preditivo de dessaturação noturna $>4 \%$ nesses pacientes. (3) Não existem dados consistentes na literatura que correlacionem o valor preditivo dos testes de função pulmonar e de valores gasométricos com a chance de dessaturação isolada durante o sono.

\section{Asma brônquica}

Há séculos é conhecido o agravamento dos sintomas da asma durante a noite, assim como a proporcionalmente maior mortalidade durante a noite e primeiras horas da manhã. Sintomas noturnos ocorrem em 60-74\% dos pacientes com asma e são marcadores de controle inadequado da doença. ${ }^{(6)}$ Mais de onze mecanismos fisiopatológicos são atribuídos a esse agravamento, sendo os mais importantes, sem excluir uma possivel associação entre os mesmos, o aumento da inflamação brônquica, alterações neuro-humorais e de receptores, refluxo gastroesofágico e SAOS. Um exemplo de associação ocorre entre os dois últimos mecanismos, quando o aumento da pressão abdominal nos períodos de apneia obstrutiva favoreceria o refluxo gastroesofágico, a hiperreatividade e a inflamação brônquicas. ${ }^{(7)}$

Já fora apontado, em um grande estudo de base populacional, o agravamento da qualidade de vida que os sintomas sugestivos de SAOS impunham aos pacientes asmáticos, e, recentemente, constatou-se um aumento dos custos de tratamento nas doenças obstrutivas crônicas quando associadas à SAOS, especialmente na coexistência de asma/ DPOC, cujos sintomas respiratórios seriam mais intensos a ponto de haver um maior número de indicações de estudos laboratoriais de sono, elevando, assim, os custos. ${ }^{(8)}$

Além de dessaturações mais graves, especialmente durante o sono REM, os pacientes asmáticos com difícil controle de asma podem ter SAOS como um mecanismo favorecendo a resistência ao tratamento. Assim, alguns autores mostraram que SAOS comprovada por polissonografia, seja em um estudo controlado, seja na pesquisa de sintomas sugestivos estudados por meio de questionários validados, pode ser muito prevalente na asma de difícil controle. ${ }^{(9)}$ Entretanto, em um estudo, não foi evidenciado que SAOS era um fator importante na asma de difícil controle, embora os autores do estudo não deixassem claro se fora utilizado um questionário de sono validado como pré-requisito para a indicação de polissonografia ou se essa foi realizada em todos os pacientes. ${ }^{(10)}$

A ativação neural da faringe por variações cíclicas de abertura e fechamento na apneia obstrutiva é atribuída como uma causa provável de agravamento do PFE na asma. Entretanto, recentemente atribuiu-se ao aumento linear da dosagem de corticosteroides inalatórios uma associação com o agravamento dos sintomas sugestivos de SAOS, sugerindo uma possível miopatia dose-dependente dos músculos da faringe, independente do controle da asma. ${ }^{(11)}$

Especula-se que SAOS pode ser inclusive um fator importante nas exacerbações de asma e 
que o uso de CPAP pode reduzir as exacerbações, melhorar a qualidade de vida e controlar/ reduzir o número de casos de asma de difícil controle, o que foi bem demonstrado em vários estudos. ${ }^{(12)} 0$ uso bem indicado e criterioso de CPAP nesses casos mostra evidências de vários efeitos benéficos interagindo no binômio asma-SAOS, como os efeitos positivos sobre a inflamação brônquica e sistêmica, a diminuição da hiper-reatividade brônquica, a melhora na arquitetura do sono, a redução do peso corpóreo, a supressão da produção de lecitina pelo tecido adiposo, a melhora na função cardíaca e a redução importante do refluxo gastroesofágico.

Asma brônquica e SAOS são dois problemas crescentes de saúde pública, com interações cada vez mais reconhecidas e com potencial para uma intervenção preventiva e de tratamento.

\section{DPOC}

Os portadores de DPOC tornam-se mais hipoxêmicos durante o sono do que durante a vigília e o exercício. ${ }^{(13)}$ Os principais mecanismos fisiopatológicos responsáveis pela hipoxemia noturna são hipoventilação, alteração da ventilação/perfusão e SAOS. (2,13) $^{2}$

Muitos portadores de DPOC apresentam uma qualidade do sono reduzida, caracterizada por menor eficiência do sono, maior latência para o início do sono e fragmentação da arquitetura do sono, independentemente da gravidade da limitação do fluxo aéreo, mas dependente da presença de SAOS. ${ }^{(14)}$ A causa é provavelmente multifatorial e inclui tosse noturna, dispneia, uso de medicações, como a teofilina, e hipoxemia recorrente.

Diversas revisões ${ }^{(2,13)}$ consideram que a hipoventilação é a principal causa de hipoxemia noturna no portador de DPOC. A hipoventilação é maior durante a fase do sono REM, uma vez que ocorre acentuada hipotonia da musculatura acessória e intercostal da respiração, resultando em uma diminuição da contribuição da caixa torácica para a ventilação. Como a hiperinsuflação pulmonar na DPOC promove a retificação das cúpulas diafragmáticas e, portanto, uma contração diafragmática ineficaz, ocorre profunda hipoventilação com hipoxemia.

Tanto SAOS como DPOC são condições clínicas comuns e, portanto, é de se esperar que a associação entre as duas patologias não seja rara. Foi cunhado o termo overlap syndrome ${ }^{(15)}$ quando as duas condições clínicas estão presentes num mesmo paciente. Essa síndrome resulta em acentuada hipoxemia relacionada ao sono com maior tendência a hipercapnia, hipertensão pulmonar e cor pulmonale, conferindo a esses pacientes um pior prognóstico. ${ }^{(16)}$

Diversos estudos situaram a prevalência da associação entre DPOC e SAOS entre 9,5-28\%.(14,16,17) Em um recente estudo multicêntrico de base populacional, foram investigadas 5.954 pessoas através de espirometria e polissonografia. ${ }^{(18)}$ Os principais achados foram os seguintes: (i) a prevalência de DPOC foi de 19\% e, entre esses, apenas 3,8\% apresentavam uma relação $\mathrm{VEF}_{1} / \mathrm{CVF}<60 \%$; (ii) entre os portadores de DPOC, a prevalência de SAOS com $1 A H>10$ eventos/h e > 15 eventos $/ \mathrm{h}$ foi de $22 \%$ e $14 \%$, respectivamente; e (iii) entre os não portadores de DPOC, a prevalência de SAOS com $1 A H>10$ eventos/h e $>15$ eventos/h foi de $29 \%$ e 19\%, respectivamente. Esses resultados não podem ser extrapolados para populações com moderada a acentuada limitação do fluxo aéreo.

As variáveis clínicas de correlação positiva para SAOS no portador de DPOC são as seguintes: ronco alto e frequente, grande circunferência do pescoço e padrão clínico-funcional definido como "polo bronquite". Vários estudos demonstraram que os achados da prova de função pulmonar não se correlacionam com SAOS. ${ }^{(14,18)}$

Portanto, as recomendações da literatura $^{(19)}$ para a indicação de polissonografia nos portadores de DPOC são: (i) sintomas sugestivos de SAOS (ronco alto, apneia presenciada por familiar e sonolência excessiva diurna); (ii) complicações da hipoxemia (por exemplo, policitemia e hipertensão pulmonar) na presença de $\mathrm{PaO}_{2}>60 \mathrm{mmHg}$; e (iii) hipertensão pulmonar desproporcional ao grau de limitação do fluxo aéreo.

Para os portadores de overlap syndrome, está indicado o uso de CPAP ou BiPAP durante o sono na presença de hipoventilação associada e consequente hipercapnia. Em um estudo recente ${ }^{(20)} 0$ aumento da sobrevida de portadores de overlap syndrome com hipoxemia diurna, em programa de oxigenoterapia e tratados com CPAP foi documentado. A sobrevida em cinco anos foi estimada em $71 \%$ e $26 \%$ nos grupos tratado e não tratado com CPAP, respectivamente. 


\section{Considerações finais}

As doenças pulmonares crônicas podem ter suas manifestações pulmonares e sistêmicas agravadas por distúrbios respiratórios do sono, em especial, as dessaturações isoladas e SAOS. 0 diagnóstico e o tratamento desses distúrbios podem interferir favoravelmente na evolução clínica das doenças pulmonares crônicas.

\section{Referências}

1. Hudgel DW, Martin RJ, Johnson B, Hill P. Mechanics of the respiratory system and breathing pattern during sleep in normal humans. J Appl Physiol. 1984;56(1):133-7.

2. McNicholas WT. Impact of sleep in COPD. Chest. 2000;117(2 Suppl):48S-53S.

3. Agarwal S, Richardson B, Krishnan V, Schneider H, Collop NA, Danoff SK. Interstitial lung disease and sleep: What is known? Sleep Med. 2009;10(9):947-51.

4. Prado GF, Allen RP, Trevisani VM, Toscano VG, Earley CJ. Sleep disruption in systemic sclerosis (scleroderma) patients: clinical and polysomnographic findings. Sleep Med. 2002;3(4):341-5.

5. Lancaster LH, Mason WR, Parnell JA, Rice TW, Loyd $\mathrm{JE}$, Milstone AP, et al. Obstructive sleep apnea is common in idiopathic pulmonary fibrosis. Chest. 2009;136(3):772-8.

6. Shigemitsu H, Afshar K. Nocturnal asthma. Curr Opin Pulm Med. 2007;13(1):49-55. Erratum in: Curr Opin Pulm Med. 2007;13(2):156-7.

7. Lewis DA. Sleep in patients with asthma and chronic obstructive pulmonary disease. Curr Opin Pulm Med. 2001;7(2):105-12.

8. Shaya FT, Lin PJ, Aljawadi MH, Scharf SM. Elevated economic burden in obstructive lung disease patients with concomitant sleep apnea syndrome. Sleep Breath. 2009;13(4):317-23.

9. Yigla M, Tov N, Solomonov A, Rubin AH, Harlev D. Difficult-to-control asthma and obstructive sleep apnea. J Asthma. 2003;40(8):865-71.
10. Araujo AC, Ferraz E, Borges Mde C, Filho JT, Vianna EO. Investigation of factors associated with difficult-tocontrol asthma. J Bras Pneumol. 2007;33(5):495-501.

11. Teodorescu M, Consens FB, Bria WF, Coffey MJ, McMorris MS, Weatherwax KJ, et al. Predictors of habitual snoring and obstructive sleep apnea risk in patients with asthma. Chest. 2009;135(5):1125-32.

12. Alkhalil M, Schulman ES, Getsy J. Obstructive sleep apnea syndrome and asthma: the role of continuous positive airway pressure treatment. Ann Allergy Asthma Immunol. 2008;101(4):350-7.

13. Mulloy E, McNicholas WT. Ventilation and gas exchange during sleep and exercise in severe COPD. Chest. 1996;109(2):387-94.

14. Santos CE, Viegas CA. Padrão do sono em pacientes portadores de doença pulmonar obstrutiva crônica e correlação entre variáveis gasométricas, espirométricas e polissonográficas. J Pneumol. 2003;29(2):69-74.

15. Flenley DC. Sleep in chronic obstructive lung disease. Clin Chest Med. 1985;6(4):651-61.

16. Chaouat A, Weitzenblum E, Krieger J, Ifoundza T, Oswald M, Kessler R. Association of chronic obstructive pulmonary disease and sleep apnea syndrome. Am J Respir Crit Care Med. 1995;151(1):82-6.

17. Cabral MM. Apnéia obstrutiva do sono em pacientes portadores de doença pulmonar obstrutiva crônica: impacto do padrão clínico-funcional [thesis]. São Paulo: Universidade de São Paulo; 2005.

18. Sanders $M H$, Newman $A B$, Haggerty $C L$, Redline $S$, Lebowitz M, Samet J, et al. Sleep and sleep-disordered breathing in adults with predominantly mild obstructive airway disease. Am J Respir Crit Care Med. 2003;167(1):714.

19. Celli BR, MacNee W; ATS/ERS Task Force. Standards for the diagnosis and treatment of patients with COPD: a summary of the ATS/ERS position paper. Eur Respir J. 2004;23(6):932-46. Erratum in: Eur Respir J. 2006;27(1):242.

20. Machado MC, Vollmer WM, Togeiro SM, Bilderback AL, Oliveira MV, Leitão FS, et al. CPAP and survival in moderate-to-severe obstructive sleep apnoea syndrome and hypoxaemic COPD. Eur Respir J. 2010;35(1):132-7.

\section{Sobre os autores}

\section{Marilia Montenegro Cabral}

Professora Adjunta. Universidade Estadual de Pernambuco; e Médica Pneumologista, Pronto Socorro Cardiológico de Pernambuco PROCAPE - Recife (PE) Brasil.

\section{Paulo de Tarso Mueller}

Professor Adjunto 1. Universidade Federal de Mato Grosso do Sul, Campo Grande (MS) Brasil.

E-mail para contato: montcabral@hotmail.com (M. Cabral) ou mpaulo@nin.ufms.br (P. Mueller) 